\title{
Flexible least squares for temporal data mining and statistical arbitrage
}

\author{
Giovanni Montana $^{\mathrm{a}, *}$, Kostas Triantafyllopoulos ${ }^{\mathrm{b}}$, Theodoros Tsagaris ${ }^{\mathrm{a}, 1}$ \\ ${ }^{a}$ Department of Mathematics, Statistics Section, Imperial College London, London SW7 2AZ, UK \\ ${ }^{\mathrm{b}}$ Department of Probability and Statistics, University of Sheffield, Sheffield S3 7RH, UK
}

\begin{abstract}
A number of recent emerging applications call for studying data streams, potentially infinite flows of information updated in realtime. When multiple co-evolving data streams are observed, an important task is to determine how these streams depend on each other, accounting for dynamic dependence patterns without imposing any restrictive probabilistic law governing this dependence. In this paper we argue that flexible least squares (FLS), a penalized version of ordinary least squares that accommodates for time-varying regression coefficients, can be deployed successfully in this context. Our motivating application is statistical arbitrage, an investment strategy that exploits patterns detected in financial data streams. We demonstrate that FLS is algebraically equivalent to the well-known Kalman filter equations, and take advantage of this equivalence to gain a better understanding of FLS and suggest a more efficient algorithm. Promising experimental results obtained from a FLS-based algorithmic trading system for the S\&P 500 Futures Index are reported.
\end{abstract}

(c) 2008 Elsevier Ltd. All rights reserved.

Keywords: Temporal data mining; Flexible least squares; Time-varying regression; Algorithmic trading system; Statistical arbitrage

\section{Introduction}

Temporal data mining is a fast-developing area concerned with processing and analyzing high-volume, highspeed data streams. A common example of data stream is a time series, a collection of univariate or multivariate measurements indexed by time. Furthermore, each record in a data stream may have a complex structure involving both continuous and discrete measurements collected in sequential order. There are several application areas in which temporal data mining tools are being increasingly used, including finance, sensor networking, security, disaster management, e-commerce and many others. In the financial arena, data streams are being monitored and explored for many different purposes such as algorithmic trading, smart order routing, real-time compliance, and fraud detec-

\footnotetext{
* Corresponding author.

E-mail address: g.montana@imperial.ac.uk (G. Montana).

1 The author is also affiliated with BlueCrest Capital Management. The views presented here reflect solely the author's opinion.
}

tion. At the core of all such applications lies the common need to make time-aware, instant, intelligent decisions that exploit, in one way or another, patterns detected in the data.

In the last decade we have seen an increasing trend by investment banks, hedge funds, and proprietary trading boutiques to systematize the trading of a variety of financial instruments. These companies resort to sophisticated trading platforms based on predictive models to transact market orders that serve specific speculative investment strategies.

Algorithmic trading, otherwise known as automated or systematic trading, refers to the use of expert systems that enter trading orders without any user intervention; these systems decide on all aspects of the order such as the timing, price, and its final quantity. They effectively implement pattern recognition methods in order to detect and exploit market inefficiencies for speculative purposes. Moreover, automated trading systems can slice a large trade automatically into several smaller trades in order to hide its impact on the market (a technique called iceberging) and lower 
trading costs. According to the financial times, the London stock exchange foresees that about $60 \%$ of all its orders in the year 2007 will be entered by algorithmic trading.

Over the years, a plethora of statistical and econometric techniques have been developed to analyze financial data De Gooijer and Hyndma, 2006. Classical time series analysis models, such as ARIMA and GARCH, as well as many other extensions and variations, are often used to obtain insights into the mechanisms that generates the observed data and make predictions Chatfield, 2004. However, in some cases, conventional time series and other predictive models may not be up to the challenges that we face when developing modern algorithmic trading systems. Firstly, as the result of developments in data collection and storage technologies, these applications generate massive amounts of data streams, thus requiring more efficient computational solutions. Such streams are delivered in real-time; as new data points become available at very high frequency, the trading system needs to quickly adjust to the new information and take almost instantaneous buying and selling decisions. Secondly, these applications are mostly exploratory in nature: they are intended to detect patterns in the data that may be continuously changing and evolving over time. Under this scenario, little prior knowledge should be injected into the models; the algorithms should require minimal assumptions about the data-generating process, as well as minimal user specification and intervention.

In this work we focus on the problem of identifying time-varying dependencies between co-evolving data streams. This task can be casted into a regression problem: at any specified point in time, the system needs to quantify to what extent a particular stream depends on a possibly large number of other explanatory streams. In algorithmic trading applications, a data stream may comprise daily or intra-day prices or returns of a stock, an index or any other financial instrument. At each time point, we assume that a target stream of interest depends linearly on a number of other streams, but the coefficients of the regression models are allowed to evolve and change smoothly over time.

The paper is organized as follows. In Section 2 we briefly review a number of common trading strategies and formulate the problem arising in statistical arbitrage, thus proving some background material and motivation for the proposed methods. The flexible least squares (FLS) methodology is introduced in Section 3 as a powerful exploratory method for temporal data mining; this method fits our purposes well because it imposes no probabilistic assumptions and relies on minimal parameter specification. In Section 4 some assumptions of the FLS method are revisited, and we establish a clear connection between FLS and the well-known Kalman filter equations. This connection sheds light on the interpretation of the model, and naturally yields a modification of the original FLS that is computationally more efficient and numerically stable. Experimental results that have been obtained using the FLS-based trading system are described in Section 5. In that section, in order to deal with the large number of predictors, we complement FLS with a feature extraction procedure that performs on-line dimensionality reduction. We conclude in Section 7 with a discussion on related work and directions for further research.

\section{A concise review of trading strategies}

Two popular trading strategies are market timing and trend following. Market timers and trend followers both attempt to profit from price movements, but they do it in different ways. A market timer forecasts the direction of an asset, going long (i.e. buying) to capture a price increase, and going short (i.e. selling) to capture a price decrease. A trend follower attempts to capture the market trends. Trends are commonly related to serial correlations in price changes; a trend is a series of asset prices that move persistently in one direction over a given time interval, where price changes exhibit positive serial correlation. A trend follower attempts to identify developing price patterns with this property and trade in the direction of the trend if and when this occurs.

Although the time-varying regression models discussed in this work may be used to implement such trading strategies, we will not discuss this further. We rather focus on statistical arbitrage, a class of strategies widely used by hedge funds or proprietary traders. The distinctive feature of such strategies is that profits can be made by exploiting statistical mispricing of one or more assets, based on the expected value of these assets.

The simplest special case of these strategies is perhaps pairs trading (see Elliott, van der Hoek, \& Malcolm, 2005; Gatev, Goetzmann, \& Rouwenhorst, 2006). In this case, two assets are initially chosen by the trader, usually based on an analysis of historical data or other financial considerations. If the two stocks appear to be tied together in the long term by some common stochastic trend, a trader can take maximum advantage from temporary deviations from this assumed equilibrium ${ }^{2}$.

A specific example will clarify this simple but effective strategy. Fig. 1 shows the historical prices of two assets, SouthWest Airlines and Exxon Mobil; we denote the two price time series by $y_{t}$ and $x_{t}$ for $t=1,2, \ldots$, respectively. Clearly, from 1997 till 2004, the two assets exhibited some dependence: their spread, defined as $s_{t}=y_{t}-x_{t}$ (plotted in the inset figure) fluctuates around a long-term average of about -20 . A trading system implementing a pairs trading strategy on these two assets would exploit temporary divergences from this market equilibrium. For instance, when the spread $s_{t}$ is greater than some predetermined positive constant $c$, the system assume that the SouthWest Airlines is overpriced and would go short on SouthWest Airlines and long on Exxon Mobil, in some predetermined ratio.

\footnotetext{
${ }^{2}$ This strategy relies on the idea of co-integration. Several applications of co-integration-based trading strategies are presented in Alexander and Dimitriu (2002) and Burgess (2003).
} 


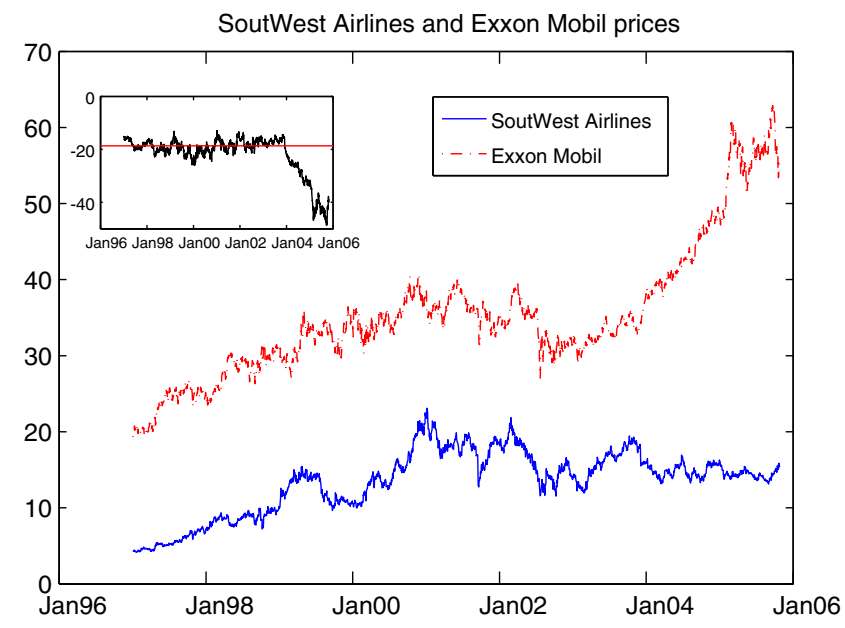

Fig. 1. Historical prices of Exxon Mobil Corporation and SouthWest Airlines for the period 1997-2007. The spread time series, reported in the inset, shows an equilibrium level between the two prices until about January 2004.

A profit is made when the prices revert back to their longterm average. Although a stable relationship between two assets may persist for quite some time, it may suddenly disappear or present itself in different patterns, such as periodic or trend patterns. In Fig. 1, for instance, the spread shows a downward trend after January 2004, which may be captured by implementing more refined models.

\subsection{A statistical arbitrage strategy}

Opportunities for pairs trading in the simple form described above are dependent upon the existence of similar pairs of assets, and thus are naturally limited. Many other variations and extensions exist that exploit temporary mispricing among securities. For instance, in index arbitrage, the investor looks for temporary discrepancies between the prices of the stocks comprising an index and the price of a futures contract ${ }^{3}$ on that index. By buying either the stocks or the futures contract and selling the other, market inefficiency can be exploited for a profit.

In this paper we adopt a simpler strategy than index arbitrage, somewhat more related to pairs trading. The trading system we develop tries to exploit discrepancies between a target asset, selected by the investor, and a paired artificial asset that reproduces the target asset. This artificial asset is represented by a data stream obtained as a linear combination of a possibly large set of explanatory streams assumed to be correlated with the target stream.

The rationale behind this approach is the following: if there is a strong association between synthetic and target assets persisting over a long period of time, this association implies that both assets react to some underlying (and unobserved) systematic component of risk that explains

\footnotetext{
${ }^{3}$ A futures contract is an obligation to buy or sell a certain underlying instrument at a specific date and price, in the future.
}

their dynamics. Such a systematic component may include all market-related sources of risk, including financial and economic factors. The objective of this approach is to neutralize all marker-related sources of risks and ultimately obtain a data stream that best represents the target-specific risk, also known as idiosyncratic risk.

Suppose that $y_{t}$ represents the data stream of the target asset, and $\hat{y}_{t}$ is the artificial asset estimated using a set of $p$ explanatory and co-evolving data streams $x_{1}, \ldots, x_{p}$, over the same time period. In this context, the artificial asset can also be interpreted as the fair price of the target asset, given all available information and market conditions. The difference $y_{t}-\hat{y}_{t}$ then represents the risk associated with the target asset only, or mispricing. Given that this construction indirectly accounts for all sources of variations due to various market-related factors, the mispricing data stream is more likely to contain predictable patterns (such as the mean-reverting behavior seen in Fig. 1) that could potentially be exploited for speculative purposes. For instance, in an analogy with the pairs trading approach, a possibly large mispricing (in absolute value) would flag a temporary inefficiency that will soon be corrected by the market. This construction crucially relies on accurately and dynamically estimating the artificial asset, and we discuss this problem next.

\section{Flexible least squares (FLS)}

The standard linear regression model involves a response variable $y_{t}$ and $p$ predictor variables $x_{1}, \ldots, x_{p}$, which usually form a predictor column vector $x_{t}=\left(x_{1 t}, \ldots, x_{p t}\right)^{\prime}$. The model postulates that $y_{t}$ can be approximated well by $x_{t}^{\prime} \beta$, where $\beta$ is a $p$-dimensional vector of regression parameters. In ordinary least square (OLS) regression, estimates $\hat{\beta}$ of the parameter vector are found as those values that minimize the cost function

$C(\beta)=\sum_{t=1}^{T}\left(y_{t}-x_{t}^{\prime} \beta\right)^{2}$

When both the response variable $y_{t}$ and the predictor vector $x_{t}$ are observations at time $t$ of co-evolving data streams, it may be possible that the linear dependence between $y_{t}$ and $x_{t}$ changes and evolves, dynamically, over time. Flexible least squares were introduced at the end of the 80 's by Tesfatsion and Kalaba (1989) as a generalization of the standard linear regression model above in order to allow for time-variant regression coefficients. Together with the usual regression assumption that

$y_{t}-x_{t}^{\prime} \beta_{t} \approx 0$

the FLS model also postulates that

$\beta_{t+1}-\beta_{t} \approx 0$

that is, the regression coefficients are now allowed to evolve slowly over time.

FLS does not require the specification of probabilistic properties for the residual error in (2). This is a favorable 
aspect of the method for applications in temporal data mining, where we are usually unable to precisely specify a model for the errors; moreover, any assumed model would not hold true at all times. We have found that FLS performs well even when assumption (3) is violated, and there are large and sudden changes from $\beta_{t-1}$ to $\beta_{t}$, for some $t$. We will illustrate this point by means of an example in the next section.

With these minimal assumptions in place, given a predictor $x_{t}$, a procedure is called for the estimation of a unique path of coefficients, $\beta_{t}=\left(\beta_{1 t}^{\prime}, \ldots, \beta_{p t}^{\prime}\right)^{\prime}$, for $t=1,2, \ldots$. The FLS approach consists of minimizing a penalized version of the OLS cost function (1), namely ${ }^{4}$

$C(\beta ; \mu)=\sum_{t=1}^{T}\left(y_{t}-x_{t}^{\prime} \beta_{t}\right)^{2}+\mu \sum_{t=1}^{T-1} \xi_{t}$

where we have defined

$\xi_{t}=\left(\beta_{t+1}-\beta_{t}\right)^{\prime}\left(\beta_{t+1}-\beta_{t}\right)$

and $\mu \geqslant 0$ is a scalar to be determined.

In their original formulation, Kalaba and Tesfatsion (1988) propose an algorithm that minimizes this cost with respect to every $\beta_{t}$ in a sequential way. They envisage a situation where all data points are stored in memory and promptly accessible, in an off-line fashion. The core of their approach is summarized in the sequel for completeness.

The smallest cost of the estimation process at time $t$ can be written recursively as

$c\left(\beta_{t+1} ; \mu\right)=\inf _{\beta_{t}}\left\{\left(y_{t}-x_{t}^{\prime} \beta_{t}\right)^{2}+\mu \xi_{t}+c\left(\beta_{t} ; \mu\right)\right\}$

Furthermore, this cost is assumed to have a quadratic form

$c\left(\beta_{t} ; \mu\right)=\beta_{t}^{\prime} Q_{t-1} \beta_{t}-2 \beta_{t}^{\prime} p_{t-1}+r_{t-1}$

where $Q_{t-1}$ and $p_{t-1}$ have dimensions $p \times p$ and $p \times 1$, respectively, and $r_{t-1}$ is a scalar. Substituting (7) into (6) and then differentiating the cost (6) with respect to $\beta_{t}$, conditioning on $\beta_{t+1}$, one obtains a recursive updating equation for the time-varying regression coefficient

$\hat{\beta}_{t}=e_{t}+M_{t} \beta_{t+1}$

with

$e_{t}=\mu^{-1} M_{t}\left(p_{t-1}+x_{t} y_{t}\right)$

$M_{t}=\mu\left(Q_{t-1}+\mu I+x_{t} x_{t}^{\prime}\right)^{-1}$

The recursions are started with some initial $Q_{0}$ and $p_{0}$. Now, using (8), the cost function can be written as

$c\left(\beta_{t+1} ; \mu\right)=\beta_{t+1}^{\prime} Q_{t+1}-2 \beta_{t+1}^{\prime} p_{t}+r_{t}$

where

\footnotetext{
${ }^{4}$ This cost function is called the incompatibility cost in Tesfatsion and Kalaba (1989).
}

$$
\begin{aligned}
& Q_{t}=\mu\left(I_{p}-M_{t}\right) \\
& p_{t}=\mu e_{t} \\
& r_{t}=r_{t-1}+y_{t}^{2}-\left(p_{t-1}+x_{t} y_{t}\right)^{\prime} e_{t}
\end{aligned}
$$

and where $I_{p}$ is the $p \times p$ identity matrix. In order to apply (8), this procedure requires all data points till time $T$ to be available, so the coefficient vector $\beta_{T}$ should be computed first. Kalaba and Tesfatsion (1988) show that the estimate of $\beta_{T}$ can be obtained sequentially as

$\hat{\beta}_{T}=\left(Q_{T-1}+x_{T} x_{T}^{\prime}\right)^{-1}\left(p_{T-1}+x_{T} y_{T}\right)$

Subsequently, (8) can be used to estimate all remaining coefficient vectors $\beta_{T-1}, \ldots, \beta_{1}$, going backwards in time.

The procedure relies on the specification of the regularization parameter $\mu \geqslant 0$; this scalar penalizes the dynamic component of the cost function (4), defined in (5), and acts as a smoothness parameter that forces the time-varying vector towards or away from the fixed-coefficient OLS solution. We prefer the alternative parameterization based on $\mu=(1-\delta) / \delta$ controlled by a scalar $\delta$ varying in the unit interval. Then, with $\delta$ set very close to 0 (corresponding to very large values of $\mu$ ), near total weight is given to minimizing the static part of the cost function (4). This is the smoothest solution and results in standard OLS estimates. As $\delta$ moves away from 0 , greater priority is given to the dynamic component of the cost, which results in time-varying estimates.

\subsection{Off-line and on-line FLS: an illustration}

As noted above, the original FLS has been introduced for situations in which all the data points are available, in batch, prior to the analysis. In contrast, we are interested in situations where each data point arrives sequentially. Each component of the $p$ dimensional vector $x_{t}$ represents a new point of a data stream, and the path of regression coefficients needs to be updated at each time step so as to incorporate the most recently acquired information. Using the FLS machinery in this setting, the estimate of $\beta_{t}$ is given recursively by

$\hat{\beta}_{t}=\left(S_{t-1}+x_{t} x_{t}^{\prime}\right)^{-1}\left(s_{t-1}+x_{t} y_{t}\right)$

where we have defined the quantities

$$
\begin{aligned}
& S_{t}=\mu\left(S_{t-1}+\mu I_{p}+x_{t} x_{t}^{\prime}\right)^{-1}\left(S_{t-1}+x_{t} x_{t}^{\prime}\right) \\
& s_{t}=\mu\left(S_{t-1}+\mu I_{p}+x_{t} x_{t}^{\prime}\right)^{-1}\left(s_{t-1}+x_{t} y_{t}\right)
\end{aligned}
$$

The recursions are initially started with some arbitrarily chosen values $S_{0}$ and $s_{0}$.

Fig. 2 illustrates how accurately the FLS algorithm recovers the path of the time-varying coefficients, in both off-line and on-line settings, for some artificially created data streams. The target stream $y_{t}$ for this example has been generated using the model

$y_{t}=x_{t} \beta_{t}+\epsilon_{t}$ 


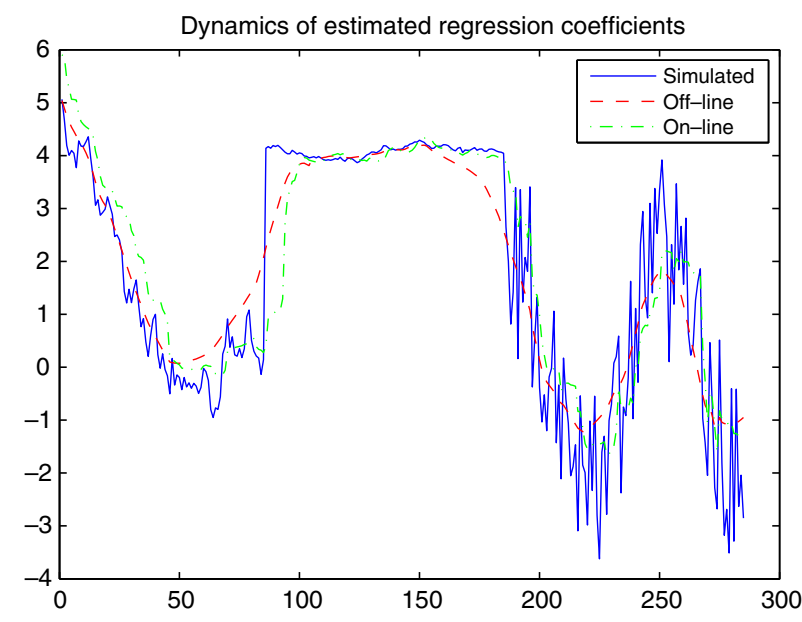

Fig. 2. Simulated versus estimated time-varying regression coefficients using FLS in both off-line and on-line mode.

where $\epsilon_{t}$ is uniformly distributed over the interval $[-2,2]$ and the explanatory stream $x_{t}$ evolves as

$x_{t}=0.8 x_{t-1}+z_{t}$

with $z_{t}$ being white noise. The regression coefficients have been generated using a slightly complex mechanism for the purpose of illustrating the flexibility of FLS. Starting with $\beta_{1}=7$, we then generate $\beta_{t}$ as

$\beta_{t}= \begin{cases}\beta_{t-1}+a_{t} & \text { for } t=2, \ldots, 99 \\ \beta_{t-1}+4 & \text { for } t=100 \\ \beta_{t-1}+b_{t} & \text { for } t=101, \ldots, 200 \\ 5 \sin (0.5 t)+c_{t} & \text { for } t=201, \ldots, 300\end{cases}$

where $a_{t}$ and $b_{t}$ are Gaussian random variables with standard deviations 0.1 and 0.001 , respectively, and $c_{t}$ is uniformly distributed over $[-2,2]$. We remark that this example features non-Gaussian error terms, as well as linear and non-linear behaviors in the dynamics of the regression coefficient, varying over time.

In this example we set $\delta=0.98$. Although such a high value of $\delta$ encourages the regression parameters to be very dynamic, the nearly constant coefficients observed between $t=101$ and $t=200$, as well as the two sudden jumps at times $t=100$ and $t=201$, are estimated well, and especially so in the on-line setting. The non-linear dynamics observed from time $t=201$ onwards is also well captured.

\section{An alternative look at FLS}

In Section 3, we have stressed that FLS relies on a quite general assumption concerning the evolution of the regression coefficients, as it only requires $\beta_{t+1}-\beta_{t}$ to be small at all times. Accordingly, assumption (3) does not imply or require that each vector $\beta_{t}$ is a random vector. Indeed, in the original work of Kalaba and Tesfatsion (1988), $\left\{\beta_{t}\right\}$ is not treated as a sequence of random variables, but rather taken as a sequence of unknown quantities to be estimated.
We ask ourselves whether we can gain a better understanding of the FLS method after assuming that the regression coefficients are indeed random vectors, without losing the generality and flexibility of the original FLS method. As it turns out, if we are willing to make such an assumption, it is possible to establish a neat algebraic correspondence between the FLS estimation equations and the well-known Kalman filter (KF) equations. This correspondence has a number of advantages. Firstly, this connection sheds light into the meaning and interpretation of the smoothing parameter $\mu$ in the cost function (4). Secondly, once the connection with KF is established, we are able to estimate the covariance matrix of the estimator of $\beta_{t}$. Furthermore, we are able to devise a more efficient version of FLS that does not require any matrix inversion. As in the original method, we restrain from imposing any specific probability distribution. The reminder of this section is dedicated to providing an alternative perspective of FLS, and deriving a clear connection between this method and the well-known Kalman filter equations.

\subsection{The state-space model}

In our formulation, the regression coefficient at time $t+1$ is modeled as a noisy version of the previous coefficient at time $t$. First, we introduce a random vector $\omega_{t}$ with zero mean and some covariance matrix $V_{\omega}$, so that

$\beta_{t+1}=\beta_{t}+\omega_{t} \quad t=0,1, \ldots, T-1$.

Then, along the same lines, we introduce a random variable $\epsilon_{t}$ having zero mean and some variance $V_{\epsilon}$, so that

$y_{t}=x_{t}^{\prime} \beta_{t}+\epsilon_{t} \quad t=1, \ldots, T$.

Eqs. (12) and (13), jointly considered, result in a linear state-space model, for which it is assumed that the innovation series $\left\{\epsilon_{t}\right\}$ and $\left\{\omega_{t}\right\}$ are mutually and individually uncorrelated, i.e. $\epsilon_{i}$ is uncorrelated of $\epsilon_{j}, \omega_{i}$ is uncorrelated of $\omega_{j}$, and $\epsilon_{k}$ is uncorrelated of $\omega_{\ell}$, for any $i \neq j$ and for any $k, \ell$. It is also assumed that for all $t, \epsilon_{t}$ and $\omega_{t}$ are uncorrelated of the initial state $\beta_{0}$. It should be emphasized again that no specific distribution assumptions for $\epsilon_{t}$ and $\omega_{t}$ have been made. We only assume that $\epsilon_{t}$ and $\omega_{t}$ attain some distributions, which we do not know. We only need to specify the first two moments of such distributions. In this sense, the only difference between the system specified by (12)(13) and FLS is the assumption of randomness of $\beta_{t}$.

\subsection{The Kalman filter}

The Kalman filter Kalman, 1960 is a powerful method for the estimation of $\beta_{t}$ in the above linear state-space model. In order to establish the connection between FLS and $\mathrm{KF}$, we derive an alternative and self-contained proof of the KF recursions that make no assumptions on the distributions of $\epsilon_{t}$ and $\omega_{t}$. We have found related proofs of such recursions that do not rely on probabilistic assumptions, as in Kalman (1960) and Eubank (2006). In comparison with 
these, we believe that our derivation is simpler and does not involve matrix inversions, which serves our purposes well.

We start with some definitions and notation. At time $t$, we denote by $\hat{\beta}_{t}$ the estimate of $\beta_{t}$ and by $\hat{y}_{t+1}=E\left(y_{t+1}\right)$ the one-step forecast of $y_{t+1}$, where $E($.) denotes expectation. The variance of $y_{t+1}$ is known as the one-step forecast variance and is denoted by $Q_{t}=\operatorname{Var}\left(y_{t+1}\right)$. The one-step forecast error is defined as $e_{t}=y_{t}-E\left(y_{t}\right)$. We also define the covariance matrix of $\beta_{t}-\hat{\beta}_{t}$ as $P_{t}$ and the covariance matrix of $\beta_{t}-\hat{\beta}_{t-1}$ as $R_{t}$ and we write $\operatorname{Cov}\left(\beta_{t}-\hat{\beta}_{t}\right)=P_{t}$ and $\operatorname{Cov}\left(\beta_{t}-\hat{\beta}_{t-1}\right)=R_{t}$. With these definitions, and assuming linearity of the system, we can see that, at time $t-1$

$$
\begin{aligned}
& R_{t}=P_{t-1}+V_{\omega} \\
& \hat{y}_{t}=x_{t}^{\prime} \hat{\beta}_{t-1} \\
& Q_{t}=x_{t}^{\prime} R_{t} x_{t}+V_{\epsilon}
\end{aligned}
$$

where $P_{t-1}$ and $\hat{\beta}_{t-1}$ are assumed known. The KF gives recursive updating equations for $P_{t}$ and $\hat{\beta}_{t}$ as functions of $P_{t-1}$ and $\hat{\beta}_{t-1}$.

Suppose we wish to obtain an estimator of $\beta_{t}$ that is linear in $y_{t}$, that is $\hat{\beta}_{t}=a_{t}+K_{t} y_{t}$, for some $a_{t}$ and $K_{t}$ (to be specified later). Then we can write

$\hat{\beta}_{t}=a_{t}^{*}+K_{t} e_{t}$

with $e_{t}=y_{t}-x_{t}^{\prime} \hat{\beta}_{t-1}$. We will show that for some $K_{t}$, if $\hat{\beta}_{t}$ is required to minimize the sum of squares

$C=\sum_{t=1}^{T}\left(y_{t}-x_{t}^{\prime} \beta_{t}\right)^{2}$

then $a_{t}^{*}=\hat{\beta}_{t-1}$. To prove this, write $Y=\left(y_{1}, \ldots, y_{T}\right)^{\prime}$, $X=\left(x_{1}^{\prime}, \ldots, x_{T}^{\prime}\right)^{\prime}, B=\left(\beta_{1}^{\prime}, \ldots, \beta_{T}^{\prime}\right)^{\prime}, \mathscr{E}=\left(e_{1}, \ldots, e_{T}\right)^{\prime}$ and

$$
K=\left(\begin{array}{llll}
K_{1} & 0 & \cdots & 0 \\
0 & K_{2} & \cdots & 0 \\
\vdots & \vdots & \ddots & \vdots \\
0 & 0 & \cdots & K_{T}
\end{array}\right)
$$

Then we can write (15) as

$C \equiv C(B)=(Y-X B)^{\prime}(Y-X B)$

and $\widehat{B}=A^{*}+K \mathscr{E}$, where $A^{*}=\left(\left(a_{1}^{*}\right)^{\prime}, \ldots,\left(a_{T}^{*}\right)^{\prime}\right)^{\prime}$. We will show that $A^{*}=B^{*}$, where $B^{*}=\left(\hat{\beta}_{0}^{\prime}, \ldots, \hat{\beta}_{T-1}^{\prime}\right)^{\prime}$. With the above $\widehat{B}$, the sum of squares can be written as

$$
\begin{aligned}
\mathscr{S}(\widehat{B})= & \left(Y-X A^{*}-X K \mathscr{E}\right)^{\prime}\left(Y-X A^{*}-X K \mathscr{E}\right) \\
= & \left(Y-X A^{*}\right)^{\prime}\left(Y-X A^{*}\right)-2\left(Y-X A^{*}\right)^{\prime} X K \mathscr{E} \\
& +\mathscr{E}^{\prime} K^{\prime} X^{\prime} X K \mathscr{E}
\end{aligned}
$$

which is minimized when $Y-X A^{*}$ is minimized or when $E\left(Y-X A^{*}\right)=0$, leading to $A^{*}=B^{*}$ as required. Thus, $a_{t}^{*}=\hat{\beta}_{t-1}$ and from (14) we have

$\hat{\beta}_{t}=\hat{\beta}_{t-1}+K_{t} e_{t}$ for some value of $K_{t}$ to be defined. From the definition of $P_{t}$, we have that

$$
\begin{aligned}
P_{t}= & \operatorname{Cov}\left(\beta_{t}-\left(\hat{\beta}_{t-1}+K_{t}\left(x_{t}^{\prime} \beta_{t}+\epsilon_{t}-x_{t}^{\prime} \hat{\beta}_{t-1}\right)\right)\right) \\
& =\operatorname{Cov}\left(\left(I_{p}-K_{t} x_{t}^{\prime}\right)\left(\beta_{t}-\hat{\beta}_{t-1}\right)-K_{t} \epsilon_{t}\right) \\
& =\left(I_{p}-K_{t} x_{t}^{\prime}\right) R_{t}\left(I_{p}-x_{t} K_{t}^{\prime}\right)+V_{\epsilon} K_{t} K_{t}^{\prime} \\
& =R_{t}-K_{t} x_{t}^{\prime} R_{t}-R_{t} x_{t} K_{t}^{\prime}+Q_{t} K_{t} K_{t}^{\prime}
\end{aligned}
$$

Now, we can choose $K_{t}$ that minimizes

$E\left(\beta_{t}-\hat{\beta}_{t}\right)^{\prime}\left(\beta_{t}-\hat{\beta}_{t}\right)$

which is the same as minimizing the trace of $P_{t}$, and thus $K_{t}$ is the solution of the matrix equation

$\frac{\partial \operatorname{trace}\left(P_{t}\right)}{\partial K_{t}}=-2\left(x_{t}^{\prime} R_{t}\right)^{\prime}+2 Q_{t} K_{t}=0$

where $\partial \operatorname{trace}\left(P_{t}\right) / \partial K_{t}$ denotes the partial derivative of the trace of $P_{t}$ with respect to $K_{t}$. Solving the above equation we obtain $K_{t}=R_{t} x_{t} / Q_{t}$. The quantity $K_{t}$, also known as the Kalman gain, is optimal in the sense that among all linear estimators $\hat{\beta}_{t}$, (16) minimizes $E\left(\beta_{t}-\hat{\beta}_{t}\right)^{\prime}\left(\beta_{t}-\hat{\beta}_{t}\right)$. With $K_{t}=R_{t} x_{t} / Q_{t}$, from (17) the minimum covariance matrix $P_{t}$ becomes

$P_{t}=R_{t}-Q_{t} K_{t} K_{t}^{\prime}$

The KF consists of Eqs. (16) and (18), together with

$$
\begin{aligned}
K_{t} & =R_{t} x_{t} / Q_{t} \\
R_{t} & =P_{t-1}+V_{\omega} \\
Q_{t} & =x_{t}^{\prime} R_{t} x_{t}+V_{\epsilon} \quad \text { and } \\
e_{t} & =y_{t}-x_{t}^{\prime} \hat{\beta}_{t-1}
\end{aligned}
$$

Initial values for $\hat{\beta}_{0}$ and $P_{0}$ have to be placed; usually we set $\hat{\beta}_{0}=0$ and $P_{0}^{-1}=0$.

Note that from the recursions of $P_{t}$ and $R_{t}$ we have

$R_{t+1}=R_{t}-Q_{t} K_{t} K_{t}^{\prime}+V_{\omega}$

\subsection{Correspondence between FLS and KF}

Traditionally, the KF equations are derived under the assumption that $\epsilon_{t}$ and $\omega_{t}$ follow the normal distribution, as in Jazwinski (1970). This stronger distributional assumption allows the derivation of the likelihood function. When the normal likelihood is available, we note that its maximization is equivalent to minimizing the quantity

$\sum_{t=1}^{T}\left(y_{t}-x_{t}^{\prime} \beta_{t}\right)^{2}+\frac{1}{V_{\omega}} \sum_{t=1}^{T-1} \xi_{t}$

with respect to $\beta_{1}, \ldots, \beta_{T}$, where $\xi_{t}$ has been defined in (5) (see Jazwinski (1970) for a proof). The above expression is exactly the cost function (4) with $\mu$ replaced by $1 / V_{\omega}$.

This correspondence can now be taken a step further: in a more general setting, where no distributional assumptions are made, it is possible to arrive to the same result. This is achieved by rearranging Eq. (9) in the form of 
(16), which is the KF estimator of $\beta_{t}$. First, note that from (10) we can write

$$
\left(S_{t-1}+x_{t} x_{t}^{\prime}\right)^{-1}=\mu S_{t}^{-1}\left(S_{t-1}+\mu I_{p}+x_{t} x_{t}^{\prime}\right)^{-1}
$$

and substituting to Eq. (9) we get $\hat{\beta}_{t}=S_{t}^{-1} s_{t}$. Thus we have

$$
\begin{aligned}
& \hat{\beta}_{t}-\hat{\beta}_{t-1}=S_{t}^{-1} s_{t}-S_{t-1}^{-1} s_{t-1} \\
& =\left(S_{t-1}+x_{t} x_{t}^{\prime}\right)^{-1}\left(s_{t-1}+x_{t} y_{t}\right)-S_{t-1}^{-1} s_{t-1} \\
& =S_{t-1}^{-1} x_{t} y_{t}-\frac{S_{t-1}^{-1} x_{t} x_{t}^{\prime} S_{t-1}^{-1}\left(s_{t-1}+x_{t} y_{t}\right)}{x_{t}^{\prime} S_{t-1}^{-1} x_{t}+1} \\
& =\frac{S_{t-1}^{-1} x_{t}}{x_{t}^{\prime} S_{t-1}^{-1} x_{t}+1}\left(y_{t} x_{t}^{\prime} S_{t-1}^{-1} x_{t}+y_{t}-x_{t}^{\prime} S_{t-1}^{-1} s_{t-1}-x_{t}^{\prime} S_{t-1}^{-1} x_{t} y_{t}\right) \\
& =\frac{S_{t-1}^{-1} x_{t}}{x_{t}^{\prime} S_{t-1}^{-1} x_{t}+1}\left(y_{t}-x_{t}^{\prime} \hat{\beta}_{t-1}\right)=K_{t} e_{t}
\end{aligned}
$$

with

$$
\begin{aligned}
K_{t} & =R_{t} x_{t} / Q_{t} \\
R_{t} & =S_{t-1}^{-1} \\
Q_{t} & =x_{t}^{\prime} R_{t} x_{t}+1 \\
V_{\epsilon} & =1
\end{aligned}
$$

It remain to prove that the recursion of $S_{t}$ as in Eq. (10) communicates with the recursion of Eq. (19), for $R_{t+1}=S_{t}^{-1}$. To end this, starting from Eq. (10) and using the matrix inversion lemma, we obtain

$$
\begin{aligned}
R_{t+1} & =S_{t}^{-1}=\mu^{-1}\left(S_{t-1}+x_{t} x_{t}^{\prime}\right)^{-1}\left(S_{t-1}+\mu I p+x_{t} x_{t}^{\prime}\right) \\
& =\mu^{-1}\left(I_{p}+\mu\left(S_{t-1}+x_{t} x_{t}^{\prime}\right)^{-1}\right) \\
& =\mu^{-1} I_{p}+\left(S_{t-1}+x_{t} x_{t}^{\prime}\right)^{-1} \\
& =S_{t-1}^{-1}-\frac{S_{t-1}^{-1} x_{t} x_{t}^{\prime} S_{t-1}^{-1}}{x_{t}^{\prime} S_{t-1}^{-1} x_{t+1}}+\mu^{-1} I_{p} \\
& =R_{t}-Q_{t} K_{t} K_{t}^{\prime}+V_{\omega}
\end{aligned}
$$

Which is the KF recursion of $R_{t}$, where

$$
V_{\omega}=\mu^{-1} I_{p}
$$

Clearly, the FLS estimator $\hat{\beta}_{t}$ of (9) is the same as the KF estimator $\hat{\beta}_{t}$ of (16). From this equivalence, it follows that $\mu=1 / V_{\omega}$, which in turn means that

$\operatorname{Cov}\left(\beta_{t+1}-\beta_{t}\right)=\frac{1}{\mu} I_{p}$

This result further clarifies the role of the smoothing parameter $\mu$ in (4). As $\mu \rightarrow \infty$, the covariance matrix of $\beta_{t+1}-\beta_{t}$ is almost zero, which means that $\beta_{t+1}=\beta_{t}$, for all $t$, reducing the model to a usual regression model with constant coefficients. In the other extreme, when $\mu \approx 0$, the covariance matrix of $\beta_{t+1}-\beta_{t}$ has very high diagonal elements (variances) and therefore the estimated $\beta_{t}$ 's fluctuate erratically.

An important computational consequence of the established correspondence between the FLS and the KF is apparent. For each time $t$, FLS requires the inversion of two matrices, namely $S_{t-1}+x_{t} x_{t}^{\prime}$ and $S_{t-1}+\mu I_{p}+x_{t} x_{t}^{\prime}$.
However, these inversions are not necessary, as it is clear by the KF that $\hat{\beta}_{t}$ can be computed by performing only matrix multiplications. This is particularly useful for temporal data mining data applications when $T$ can be infinite and $p$ very large.

It is interesting to note how the two procedures arrive to the same solution, although they are based on quite different principles. On one hand, FLS merely solves an optimization problem, as it minimizes the cost function $C(\mu)$ of (4). On the other hand, KF performs two steps: first, all linear estimators are restricted to forms of (16), for any parameter vector $K_{t}$; in the second step, $K_{t}$ is optimized so that it minimizes $P_{t}$, the covariance matrix of $\beta_{t}-\hat{\beta}_{t}$. This matrix, known as the error matrix of $\beta_{t}$, gives a measure of the uncertainty of the estimation of $\beta_{t}$.

The relationship between FLS and KF has important implications for both methods. For FLS, it suggests that the regression coefficients can be learned from the data in a recursive way without the need of performing matrix inversions; also, the error matrix $P_{t}$ is routinely available to us. For KF, we have proved that the estimator $\hat{\beta}_{t}$ minimizes the cost function $C(\mu)=C\left(1 / V_{\omega}\right)$ when only the mean and the variance of the innovations $\epsilon_{t}$ and $\omega_{t}$ are specified, without assuming these errors to be normally distributed.

\section{An FLS-based algorithmic trading system}

\subsection{Data description}

We have developed a statistical arbitrage system that trades S\&P 500 stock-index futures contracts. The underlying instrument in this case is the S\&P 500 Price Index, a world renowned index of 500 US equities with minimum capitalization of $\$ 4$ billion each; this index is a leading market indicator, and is often used as a gauge of portfolio performance. The constituents of this index are highly traded by traditional asset management firms and proprietary desks worldwide. The data stream for the S\&P 500 Futures Index, our target asset, has been kindly made available by

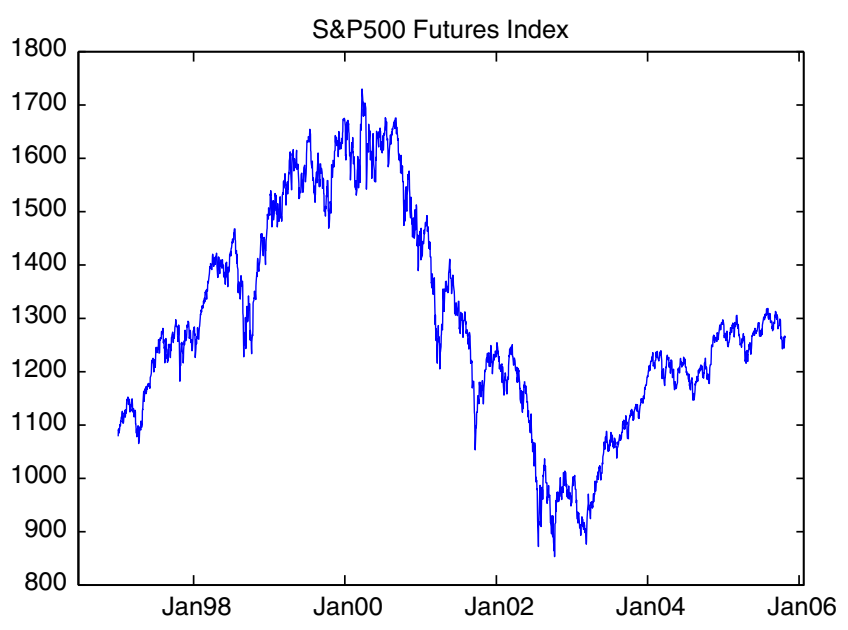

Fig. 3. S\&P 500 Futures Index for the available 9-years period. 
BlueCrest Capital Management, and covers a period of about 9 years, from 02/01/1997 to 25/10/2005 (see Fig. 3). Our explanatory data streams are taken to be a subset of all constituents of the underlying S\&P 500 Price Index. The constituents list was acquired from the Standard \& Poor's web site as of 1st of March 2007, whereas the constituents data streams were downloaded from Yahoo! financial. The constituents of the S\&P index are added and deleted frequently on the basis of the characteristics of the index. For our experiments, we have selected a time-invariant subset of 432 stocks, namely all the constituents whose historical data is available over the entire 1997-2005 period.

The system thus monitors 433 co-evolving data streams comprising one target asset and 432 explanatory streams. All raw prices are pre-processed in several ways: data adjustments are made for discontinuities relating to stock splits, bonus issues, and other financial events; missing observations are filled in using the most recent data points; finally, prices are transformed into log-returns. At each time $t>1$, the log-return for asset $i$ is defined as

$r_{i t}=\log p_{i t}-\log p_{i(t-1)} \quad i=1, \ldots, 432$

where $p_{i t}$ is the observed price of asset $i$ at time $t$. Taking returns provides a more convenient representation of the assets, as it makes different prices directly comparable and center them around zero. We collect all explanatory assets available at time $t$ in a column vector $r_{t}$. Analogously, we denote by $a_{t}$ the log-return of the S\&P 500 Futures Index at time $t$.

\subsection{Incremental $S V D$ for dimensionality reduction}

When the dimensionality of the regression model is large, as in our application, the model might suffer from multicollinearity. Moreover, in real-world trading applications using high frequency data, the regression model generating trading signals need to be updated quickly as new information is acquired. A much smaller set of explanatory streams would achieve remarkable computational speedups. In order to address all these issues, we implement on-line feature extraction by reducing the dimensionality in the space of explanatory streams.

Suppose that $R_{t}=E\left(r_{t} r_{t}^{\prime}\right)$ is the unknown population covariance matrix of the explanatory streams, with data available up to time $t=1, \ldots, T$. The algorithm proposed by Weng, Zhang, and Hwang (2003) provides an efficient procedure to incrementally update the eigenvectors of the $R_{t}$ matrix as new data are made available at time $t+1$. In turn, this procedure allows us to extract the first few principal components of the explanatory data streams in real-time, and effectively perform incremental dimensionality reduction.

A brief outline of the procedure suggested by Weng et al. (2003) is provided in the sequel. First, note that the eigenvector $g_{t}$ of $R_{t}$ satisfies the characteristic equation $h_{t}=\lambda_{t} g_{t}=R_{t} g_{t}$

where $\lambda_{t}$ is the corresponding eigenvalue. Let us call $\hat{h}_{t}$ the current estimate of $h_{t}$ using all the data up to time $t$ $(t=1, \ldots, T)$. We can write the above characteristic equation in matrix form as

$h=\left(\begin{array}{l}h_{1} \\ \vdots \\ h_{T}\end{array}\right)=\left(\begin{array}{ccc}R_{1} & \cdots & 0 \\ \vdots & \ddots & \vdots \\ 0 & \cdots & R_{T}\end{array}\right)\left(\begin{array}{l}g_{1} \\ \vdots \\ g_{T}\end{array}\right)=R g$

and then, noting that

$\frac{h_{1}+\cdots+h_{T}}{T}=\frac{1}{T}(1, \ldots, 1)^{\prime} h=\frac{1}{T}\left(R_{1}, \ldots, R_{T}\right) g=\frac{1}{T} \sum_{i=1}^{T} R_{i} g_{i}$

the estimate $\widehat{h}_{T}$ is obtained by $\widehat{h}_{T}=\left(h_{1}+\cdots+h_{T}\right) / T$ by substituting $R_{i}$ by $r_{i} r_{i}^{\prime}$. This leads to

$\hat{h}_{t}=\frac{1}{t} \sum_{i=1}^{t} r_{i} r_{i}^{\prime} g_{i}$

which is the incremental average of $r_{i} r_{i}^{\prime} g_{i}$, where $r_{i} r_{i}^{\prime}$ accounts for the contribution to the estimate of $R_{i}$ at point $i$.

Observing that $g_{t}=h_{t} /\left\|h_{t}\right\|$, an obvious choice is to estimate $g_{t}$ as $\hat{h}_{t-1} /\left\|\hat{h}_{t-1}\right\|$; in this setting, $\hat{h}_{0}$ is initialized by equating it to $r_{1}$, the first direction of data spread. After plugging in this estimator in (21), we obtain

$h_{t}=\frac{1}{t} \sum_{i=1}^{t} r_{i} r_{i}^{\prime} \frac{\widehat{h}_{i-1}}{\left\|\widehat{h}_{i-1}\right\|}$

In a on-line setting, we need a recursive expression for $\widehat{h}_{t}$. Eq. (22) can be rearranged to obtain an equivalent expression that only uses $\widehat{h}_{t-1}$ and the most recent data point $r_{t}$,

$$
\begin{aligned}
\hat{h}_{t} & =\frac{1}{t} \sum_{i=1}^{t-1} r_{i} r_{i}^{\prime} \frac{\hat{h}_{i-1}}{\left\|\hat{h}_{i-1}\right\|}+\frac{1}{t} r_{t} r_{t}^{\prime} \frac{\hat{h}_{t-1}}{\left\|\hat{h}_{t-1}\right\|} \\
& =\frac{t-1}{t} \hat{h}_{t-1}+\frac{1}{t} r_{t} r_{t}^{\prime} \frac{\hat{h}_{t-1}}{\left\|\hat{h}_{t-1}\right\|}
\end{aligned}
$$

The weights $(t-1) / t$ and $1 / t$ control the influence of old values in determining the current estimates. Full details related to the computation of the subsequent eigenvectors can be found in the contribution of Weng et al. (2003).

In our application, we have used data points from 02/ $01 / 1997$ till $01 / 11 / 2000$ as a training set to obtain stable estimates of the first few dominant eigenvectors. Therefore, data points prior to $01 / 11 / 2000$ will be excluded from the experimental results.

\subsection{Trading rule}

The trade unit for S\&P 500 Futures Index is set by the Chicago Mercantile Exchange (CME) to \$250 multiplied by the current S\&P 500 Price Index, $p_{t}$. Accordingly, we denote the trade unit expressed in monetary terms as $C_{t}=250 p_{t}$, which also gives the contract value at time $t$. For instance, if the current stock-index price 

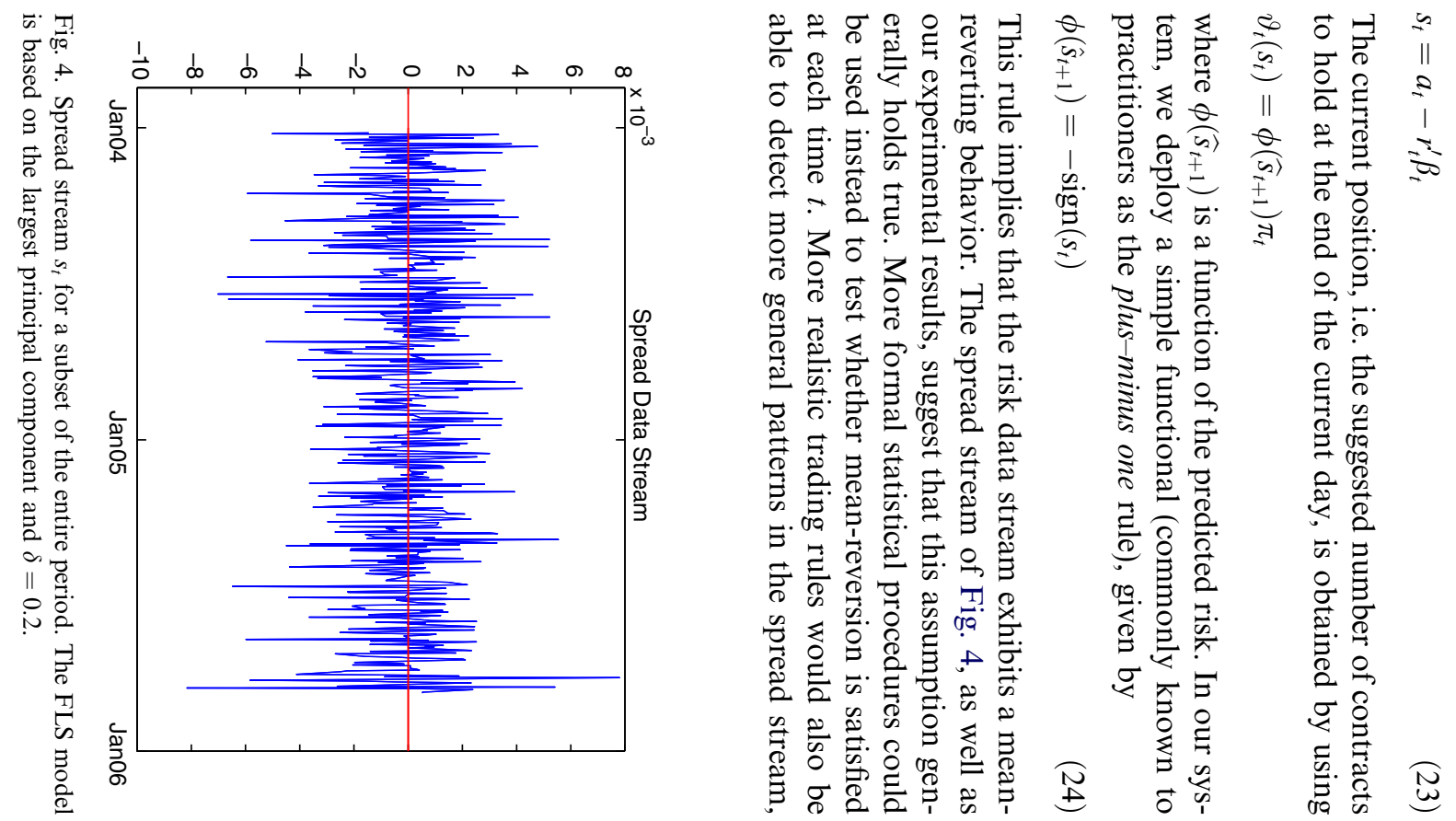

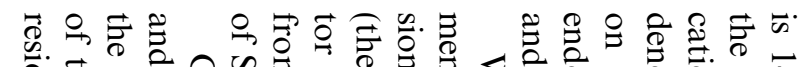

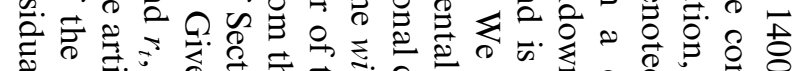

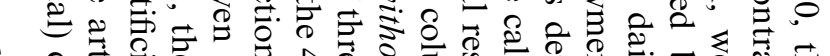

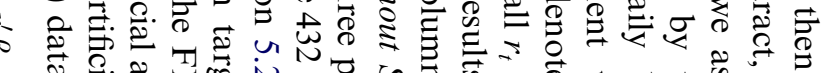

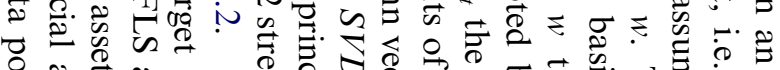
:

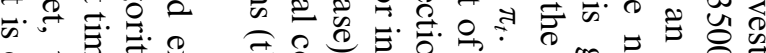

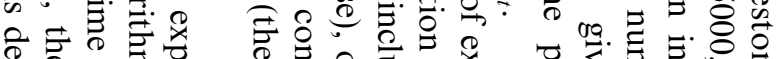

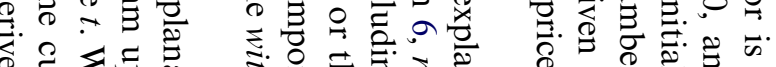

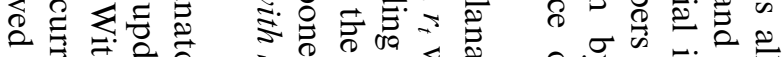

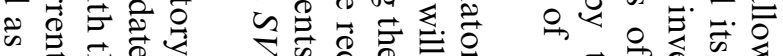
$=\vec{\sigma} \&$ is ㄹ. ค. $\stackrel{2}{\Xi}$ (1)

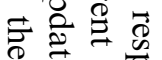

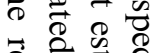
$\checkmark$ क 20

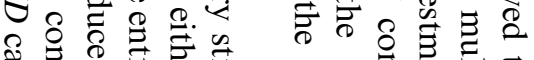

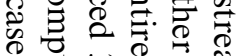

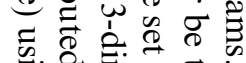

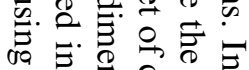
$\vec{\nabla} 8$.

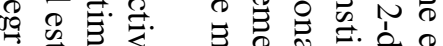
류류

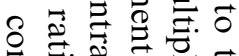

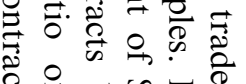
용 $\sigma \circ$ $\approx \vec{E} \cdot \stackrel{\oplus}{\leftrightarrows} \cdot \overrightarrow{8}$ \& $\vec{\sigma}$ 它.

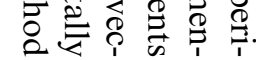

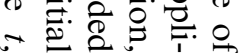

Table 1

Experimental results obtained using the statistical arbitrage system of Section 5 on 9-years of S\&P 500 Future Index

\begin{tabular}{|c|c|c|c|c|c|c|c|c|c|c|c|c|c|c|c|c|c|c|c|c|}
\hline$\delta$ & $\%$ gain & & $\%$ loss & & MDD & & $\% \mathrm{WT}$ & & $\%$ LT & & Ann.R. & & Ann.V. & & Sharpe & & In-MS & & Out-N & $\mathrm{SE}^{*}$ \\
\hline 0.01 & 0.786 & 0.773 & -0.802 & -0.817 & 31.809 & 28.529 & 47.886 & 48.732 & 43.659 & 42.813 & 6.559 & 6.728 & 16.393 & 16.393 & 0.400 & 0.410 & 0.159 & 0.019 & 2.328 & 2.311 \\
\hline 0.10 & 0.797 & 0.788 & -0.789 & -0.799 & 31.569 & 38.770 & 48.194 & 46.887 & 43.351 & 44.658 & 10.610 & 3.118 & 16.384 & 16.397 & 0.648 & 0.190 & 0.153 & 0.003 & 2.270 & 2.329 \\
\hline 0.20 & 0.803 & 0.792 & -0.783 & -0.795 & 28.616 & 34.777 & 48.501 & 46.810 & 43.044 & 44.735 & 13.175 & 3.739 & 16.377 & 16.396 & 0.804 & 0.228 & 0.149 & 0.001 & 2.243 & 2.333 \\
\hline 0.30 & 0.801 & 0.782 & -0.785 & -0.805 & 26.645 & 31.541 & 48.578 & 46.964 & 42.967 & 44.581 & 13.080 & 2.115 & 16.377 & 16.398 & 0.799 & 0.129 & 0.147 & 0.000 & 2.229 & 2.335 \\
\hline 0.40 & 0.797 & 0.789 & -0.789 & -0.798 & 30.201 & 28.432 & 48.117 & 46.887 & 43.428 & 44.658 & 10.287 & 3.365 & 16.385 & 16.397 & 0.628 & 0.205 & 0.144 & 0.000 & 2.221 & 2.336 \\
\hline 0.50 & 0.788 & 0.789 & -0.800 & -0.798 & 29.608 & 29.157 & 48.424 & 46.887 & 43.121 & 44.658 & 9.253 & 3.356 & 16.388 & 16.397 & 0.565 & 0.205 & 0.142 & 0.000 & 2.214 & 2.336 \\
\hline 0.60 & 0.789 & 0.788 & -0.799 & -0.800 & 30.457 & 32.752 & 48.655 & 46.656 & 42.890 & 44.889 & 10.381 & 2.139 & 16.385 & 16.398 & 0.634 & 0.130 & 0.140 & 0.000 & 2.210 & 2.337 \\
\hline 0.70 & 0.787 & 0.781 & -0.801 & -0.806 & 30.457 & 36.569 & 48.886 & 46.272 & 42.660 & 45.273 & 10.819 & -0.950 & 16.384 & 16.398 & 0.660 & -0.058 & 0.137 & 0.000 & 2.206 & 2.337 \\
\hline 0.80 & 0.789 & 0.782 & -0.798 & -0.806 & 33.208 & 34.217 & 48.732 & 46.580 & 42.813 & 44.965 & 10.794 & 0.490 & 16.384 & 16.398 & 0.659 & 0.030 & 0.134 & 0.000 & 2.202 & 2.338 \\
\hline 0.90 & 0.791 & 0.786 & -0.796 & -0.801 & 36.795 & 32.828 & 48.194 & 46.503 & 43.351 & 45.042 & 9.074 & 1.144 & 16.388 & 16.398 & 0.554 & 0.070 & 0.128 & 0.000 & 2.199 & 2.338 \\
\hline 0.99 & 0.800 & 0.787 & -0.787 & -0.800 & 32.782 & 33.773 & 47.809 & 46.580 & 43.736 & 44.965 & 9.587 & 1.689 & 16.387 & 16.398 & 0.585 & 0.103 & 0.102 & 0.000 & 2.205 & 2.338 \\
\hline
\end{tabular}

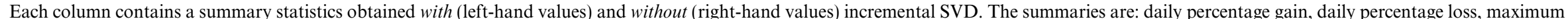

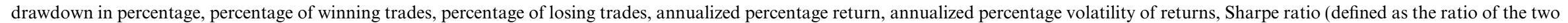
previous quantities), in-sample MSE and out-sample MSE.

To be multiplied by $10 e 5$. 
and should take into consideration the uncertainty associated with the presence of such patterns, as well the history of previous trading decisions.

Having obtained the number of contracts to hold, the daily order size is given by

$\varphi_{t}=\vartheta_{t}\left(s_{t}\right)-\vartheta_{t-1}\left(s_{t}\right)$

rounded to the nearest integer. The trading systems buys or sells daily in order to maintain the suggested number of contracts. The monetary return realized by the system at each time $t$ is given by

$f_{t}=250\left(p_{t}-p_{t-1}\right) \vartheta_{t-1}\left(s_{t}\right)$

\section{Experimental results}

In this section we report on experimental results obtained from the simple FLS-based trading system. We have tested the system using a grid of values for the smoothing parameter $\delta$ described in Section 3, to understand the effect of its specification. Table 1 shows a number of financial performance indicators, as well as a measure of goodness of fit, with and without incremental SVD.

The most important financial indicator is the Sharpe ratio, defined as the ratio between the average monetary returns and its standard deviation. It gives a measure of the mean excess return per unit of risk; values greater than 0.5 are considered very satisfactory, given that our strategy trades one single asset only. Another financial indicator reported here is the maximum drawdown, the largest movement from peak to bottom of the cumulative monetary return, reported as percentage. The mean square error (MSE) has been computed both in sample and out of sample.

Fig. 5 shows gross percentage returns over the initial endowment for the constituent set, $f_{t} / w$, obtained using three different systems: FLS-based system with incremental

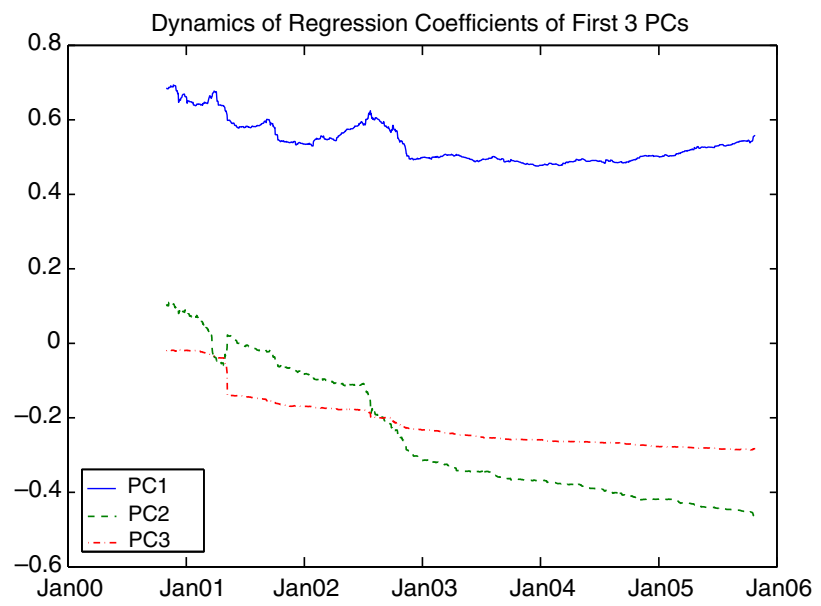

Fig. 6. Dynamics of FLS-estimated regression coefficients associated to the first three principal components, with $\delta=0.2$.

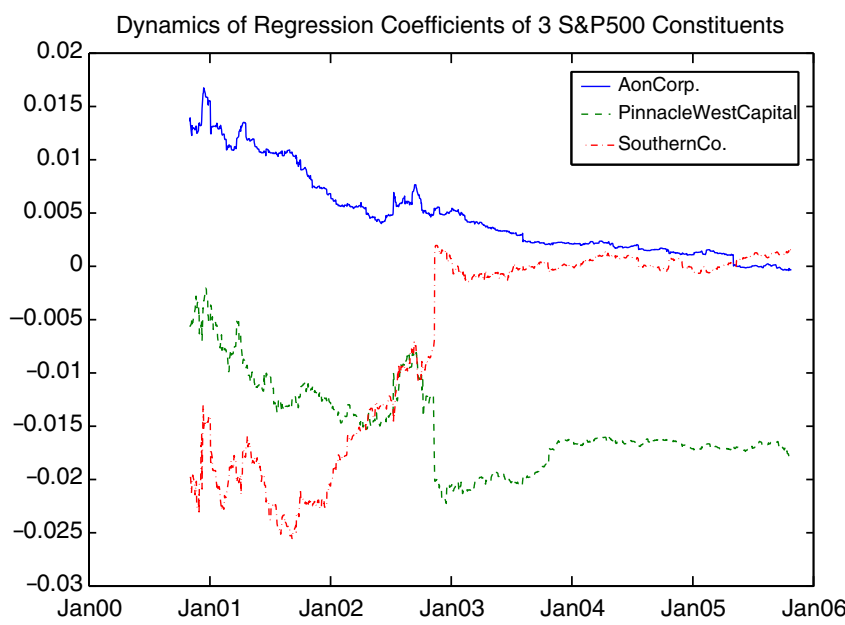

Fig. 7. Dynamics of FLS-estimated regression coefficients associated to three constituents of the index, with $\delta=0.2$.

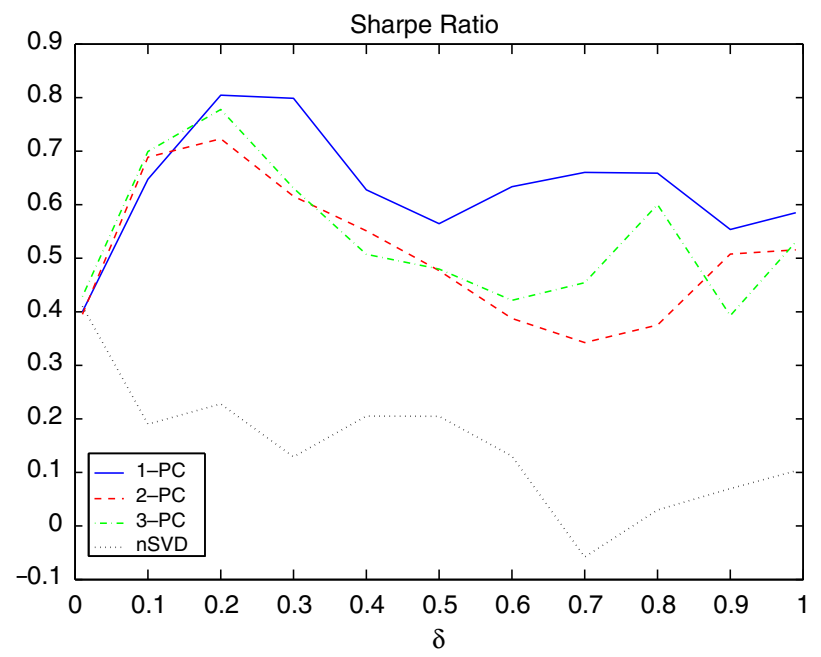

Fig. 8. Sharpe ratio as function of $\delta$.
Fig. 5. Gross profits and losses for three competing systems: FLS-based on SVD (using $\delta=0.2$ ), FLS-based on all explanatory streams (using

$\delta=0.2$ ) and a buy-and-hold strategy.

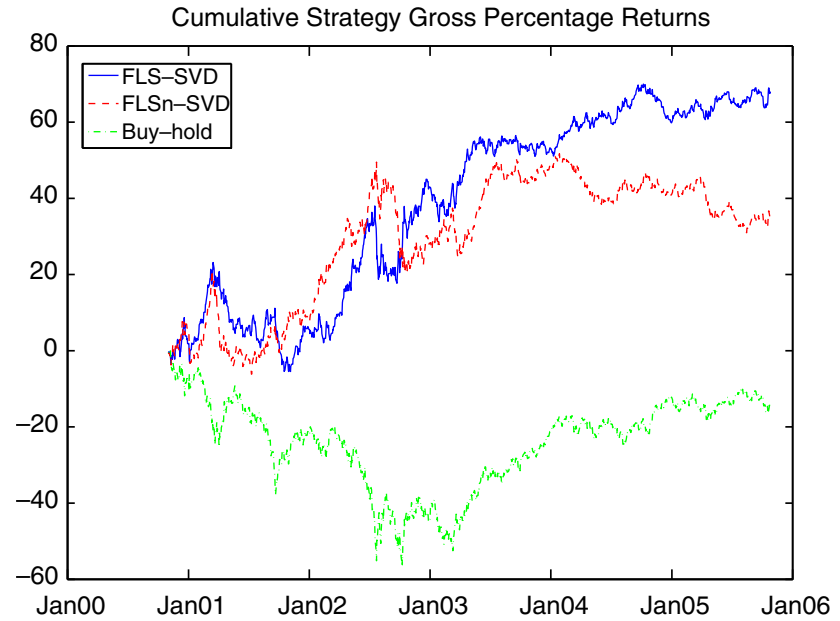


SVD (using only the largest principal component), FLSbased system without SVD, and a buy-hold strategy. Buy-hold strategies are typical of asset management firms and pension funds; the investor buys a number of contracts and holds them throughout the investment period in question. Clearly, the FLS-based systems outperforms the index and make a steady gross profit over time. The assumption of non existence of transactions costs, although simplistic, is not particularly restrictive, as we expect that this strategy will not be dominated by cost, given that new transactions are made only daily. Moreover, we assume that the initial endowment remains constant throughout the back-testing period, which has an economic meaning that the investor/agent consumes any capital gain, as soon as is earned.

Finally, Fig. 6 shows the estimated time-varying regression coefficients of the three first principal components, and Fig. 7 shows coefficients of three constituent assets when no SVD has been applied. The coefficients associated to the first component change very little over the 9 years period, whereas the coefficients for the two other components smoothly decrease over time, with some quite abrupt jumps in the initial months of 2001. As we can see from Table 1, a fairly large value of $\delta=0.2$ gives optimal results and reinforces the merits of time-varying regression in this context (see Fig. 8).

\section{Conclusions}

We have argued that the FLS method for regression with time-varying coefficients lends itself to a useful temporal data mining tool. We have derived a clear connection between FLS and Kalman filter equations, and have demonstrated how this link enhances interpretation of the smoothing parameter featuring in cost function that FLS minimizes, and naturally leads to a more efficient algorithm. Finally, we have shown how FLS can be employed as a building-block of an algorithmic trading system.

There are several aspects of the simple system presented in Section 5 that can be further improved upon, and the remainder of this discussion points to a few general directions and related work that we intend to explore in the future.

The problem of feature selection is an important one. In Section 5 the system relies on a set of 432 constituents of the S\&P 500 Price Index under the assumption that they explain well the daily movements in the target asset. These explanatory data streams could be selected automatically, perhaps even dynamically, from a very large basket of streams, on the basis of they similarity to the target asset. This line of investigation relates to the correlation detection problem for data streams, a well-studied and recurrent issue in temporal data mining. For instance, Guha, Gunopulos, and Koudas (2003) propose an algorithm that aims at detecting linear correlation between multiple streams. At the core of their approach is a technique for approximating the SVD of a large matrix by using a (random) matrix of smaller size, at a given accuracy level; the SVD is then peri- odically and randomly re-computed over time, as more data points arrive. The SPIRIT system for streaming pattern detection of Papadimitriou, Sun, and Faloutsos (2005) and Sun, Papadimitriou, and Faloutsos (2006) incrementally finds correlations and hidden variables summarising the key trends in the entire stream collection.

Of course, deciding on what similarity measure to adopt in order to measure how close explanatory and target assets are is not an easy task, and is indeed a much debated issue (see, for instance, Gavrilov, Anguelov, Indyk, \& Motwani (2000)). For instance, Shasha and Zhu (2004) adopt a sliding window model and the Euclidean distance as a measure of similarity among streams. Their StatStream system can be used to detect pairs of financial time series with high correlation, among many available data streams. Cole, Shasha, and Zhao (2005) combine several techniques (random projections, grid structures, and others) in order to compute Pearson correlation coefficients between data streams. Other measures, such as dynamic time warping, have also been suggested Capitani and Ciaccia, 2005.

Real-time feature selection can be complemented by feature extraction. In our system, for instance, we incrementally reduce the original space of 432 explanatory streams to a handful of dimensions using an on-line version of SVD. Other dynamic dimensionality reduction models, such as incremental independent component analysis Basalyga and Rattray, 2004 or non-linear manifold learning Law, Zhang, and Jain, 2004, as well as on-line clustering methods, would offer potentially useful alternatives.

Our simulation results have shown gross monetary results, and we have assumed that transaction costs are negligible. Better trading rules that explicitly model the mean-reverting behavior (or other patterns) of the spread data stream and account for transaction costs, as in Carcano, Falbo, and Stefani (2005), can be considered. The trading rule can also be modified so that trades are placed only when the spread is, in absolute value, greater than a certain threshold determined in order to maximize profits, as in Vidyamurthy (2004). In a realistic scenario, rather than trading one asset only, the investor would build a portfolio of models; the resulting system may be optimized using measures that capture both the forecasting and financial capabilities of the system, as in Towers and Burgess (2001).

Finally, we point out that the FLS method can potentially be used in other settings and applications, such as predicting co-evolving data streams with missing or delayed observations, as in Yi et al. (2000), and for outlier and fraud detection, as in Adams, Hand, Montana, and Weston (2006).

\section{Acknowledgement}

We would like to thank David Hand for helpful comments on an earlier draft of the paper. 


\section{References}

Adams, N., Hand, D.J., Montana, G., Weston, J.D., \& Whitrow. C.W. (2006). Fraud detection in consumer credit. In UK KDD Symposium (UKKDD'06).

Alexander, C., \& Dimitriu, A. (2002). The cointegration alpha: Enhanced index tracking and long-short equity market neutral strategies. Technical report, ISMA Center, University of Reading.

Basalyga, G., \& Rattray, M. (2004). Statistical dynamics of on-line independent component analysis. The Journal of Machine Learning Research, 4, 1393-1410.

Burgess, A. N. (2003). Applied quantitative methods for trading and investment. Chapter using cointegration to hedge and trade international equities (pp. 41-69), Wiley Finance.

Capitani, P., \& Ciaccia, P. (2005). Efficiently and accurately comparing real-valued data streams. In thirteenth Italian national conference on advanced data base systems (SEBD 2005).

Carcano, G., Falbo, P., \& Stefani, S. (2005). Speculative trading in mean reverting markets. European Journal of Operational Research, 163, 132-144.

Chatfield, C. (2004). The analysis of time series: An introduction (sixth ed.). New York: Chapman and Hall.

Cole, R., Shasha, D., \& Zhao, X. (2005). Fast window correlations over uncooperative time series. In Proceeding of the eleventh ACM SIGKDD international conference on knowledge discovery in data mining (pp. 743-749).

De Gooijer, J. G., \& Hyndma, R. J. (2006). 25 years of time series forecasting. International Journal of Forecasting, 22, 443-473.

Elliott, R. J., van der Hoek, J., \& Malcolm, W. P. (2005). Pairs trading. Quantitative Finance, 271-276.

Eubank, R. L. (2006). A Kalman Filter Primer. New York: Chapman and Hall.

Gatev, E., Goetzmann, W. N., \& Rouwenhorst, K. G. (2006). Pairs trading: Performance of a relative-value arbitrage rule. Review of Financial Studies, 19(3), 797-827.

Gavrilov, M., Anguelov, D., Indyk, P., \& Motwani, R. (2000). Mining the stock market: which measure is best? In Proceedings of the sixth ACM
SIGKDD international conference on knowledge discovery and data mining.

Guha, S., Gunopulos, D., \& Koudas, N. (2003). Correlating synchronous and asynchronous data streams. In Proceedings of the ninth ACM SIGKDD international conference on knowledge discovery and data mining (pp. 529-534).

Jazwinski, A. H. (1970). Stochastic processes and filtering theory. New York: Academic Press

Kalaba, R., \& Tesfatsion, L. (1988). The flexible least squares approach to time-varying linear regression. Journal of Economic Dynamics and Control, 12(1), 43-48.

Kalman, R. E. (1960). A new approach to linear filtering and prediction problems. Journal of Basic Engineering, 82, 35-45.

Law, M.H.C., Zhang, N., \& Jain, A. (2004). Nonlinear manifold learning for data stream. In Proceedings of SIAM international conference on data mining.

Papadimitriou, S., Sun, J., \& Faloutsos, C. (2005). Streaming pattern discovery in multiple time-series. In Proceedings of the 31st international conference on very large data bases (pp. 697-708).

Shasha, D., \& Zhu, Y. (2004). High performance discovery in time series. Techniques and cases studies. Springer.

Sun, J., Papadimitriou, S., \& Faloutsos, C. (2006). Distributed pattern discovery in multiple streams. In Proceedings of the Pacific-Asia conference on knowledge discovery and data mining (PAKDD).

Tesfatsion, L., \& Kalaba, R. (1989). Time-varying linear regression via flexible least squares. Computers \& Mathematics with Applications, 17(8-9), 1215-1245.

Towers, N., \& Burgess, N. (2001). Developments in forecast combination and portfolio choice. Chapter a meta-parameter approach to the construction of forecasting models for trading systems (pp. 27-44), Wiley.

Vidyamurthy, G. (2004). Pairs trading, Wiley Finance.

Weng, J., Zhang, Y., \& Hwang, W. S. (2003). Candid covariance-free incremental principal component analysis. IEEE Transactions on Pattern Analysis and Machine Intelligence, 25(8), 1034-1040.

Yi, B., et al. (2000). Online data mining for co-evolving time sequences. In Proceedings of the sixteenth international conference on data engineering (pp. 13-22) 